\section{Denosshan Sri}

\section{A telephone survey of intensive care unit handover practices in the UK}

Accepted: 12 July 2012

Published online: 10 August 2012

(C) Copyright jointly held by Springer and ESICM 2012

Dear Editor,

Rhodes et al. [1] discuss the importance of a standardized

interdepartmental handover in order to improve the safety and quality of care for critically ill patients. We feel that intradepartmental handover is as crucial.

It is widely accepted that deficiencies in handover processes exist. Studies have looked at the need for structured handover, the advantages of using standardised checklists and protocols and various other aspects of handover [2-5]. We conducted a telephone survey of hospital intensive care units (ICUs) in South and East England and Wales with the aim of investigating the consistency of handover practices among doctors and nurses. We derived a standard set of questions regarding doctor and nursing handover practice from the literature.

Of the 134 hospital ICUs surveyed, we obtained a $72 \%$ response rate $(1 / 3$ of the units nationally), and of these $75 \%$ had a combined ICU/HDU (high dependency unit) and $74 \%$ were DGHs (district general hospitals). Amongst the 1,153 beds in the study, 399 were designated ICU, 281
HDU and the remaining 473 were undesignated. Eighty seven per cent $(20 / 23)$ of teaching hospitals described themselves as combined units, whilst 56/74 (76 \%) of DGHs were combined units.

Compared to doctors' handover, nursing handover was a more regimented process with 93 units having a central nursing handover for all staff, followed by a 1:1 handover at the bedside. Almost half of the units use a printed patient list. Proformas are more widely used in nursing handovers than doctors' handovers (73 and 56 units, respectively). Twenty one ICUs include the care bundle with the nursing handover. Ninety five $(98 \%)$ ICUs have a formal doctors' handover each morning. The majority of doctors' handovers $(68 \%)$ occur at a central location, and $76 \%$ of units use a printed patient list.

The mean length of nursing handover is 24 min (standard deviation, SD 8.4), while the mean length of handover between doctors is $29 \mathrm{~min}$ (SD 13.1). Interestingly, we found that unit size was almost unrelated to the nursing handover length $\left(r^{2}=0.0025 ; p=0.312\right)$, but had some correlation with the length of doctor handover $\left(r^{2}=0.0456\right.$; $p=0.018$ ). However, there are other variables beyond unit size which must be taken into account, such as patient condition and familiarity.

This was an observational study of handover procedures in ICUs which was designed to assess practices, not evaluate quality, as there is not yet a consensus in the literature as to the best way to hand over patients. Our survey confirms that formal handovers are used in almost all units and highlights some interesting variations between the handovers of nurses and doctors.

We agree with Rhodes et al. [1] that handover documentation should ideally form part of the routine patient record. It will be interesting to see, as is the case in some units we surveyed, whether this might become universal practice in the future and form part of the patients' medicolegal record of their in-patient stay.

Acknowledgments Dr L. Tyson, $\mathrm{Dr}$ R. Stewart, Dr K. Williams and Dr V. Prasad, Lister Hospital, ITU Department, Coreys Mill Lane, Stevenage, UK.

\section{References}

1. Rhodes A, Moreno RP, Azoulay E, Capuzzo M, Chiche JD, Eddleston J, Endacott R, Ferdinande P, Flaatten H, Guidet B, Kuhlen R, León-Gil C, Martin Delgado MC, Metnitz PG, Soares M, Sprung CL, Timsit JF, Valentin A, Task Force on Safety and Quality of European Society of Intensive Care Medicine (ESICM) (2012) Prospectively defined indicators to improve the safety and quality of care for critically ill patients: a report from the Task Force on Safety and Quality of the European Society of Intensive Care Medicine (ESICM). Intensive Care Med 38:598-605

2. Craig R, Moxey L, Young D, Spenceley NS, Davidson MG (2012) Strengthening handover communication in pediatric cardiac intensive care. Paediatr Anaesth 22:393-399

3. Joy BF, Elliott E, Hardy C, Sullivan C, Backer CL, Kane JM (2011) Standardized multidisciplinary protocol improves handover of cardiac surgery patients to the intensive care unit. Pediatr Crit Care Med 12:304-308

4. Kalkman CJ (2010) Handover in the perioperative care process. Curr Opin Anaesthesiol 23:749-753

5. Pickering BW, Hurley K, Marsh B (2009) Identification of patient information corruption in the intensive care unit: using a scoring tool to direct quality improvements in handover. Crit Care Med 37:2905-2912

D. Sri $(\square)$

Lister Hospital, Stevenage,

Hertfordshire, UK

e-mail: denosshansri@gmail.com 